\title{
Turbulent Convection Insights from Small-Scale Thermal Forcing with Zero Net Heat Flux at a Horizontal Boundary
}

\author{
Ross W. Griffiths* and Bishakhdatta Gayen \\ Research School of Earth Sciences, Australian National University, Canberra ACT 0200, Australia
}

(Received 20 July 2015; published 9 November 2015)

\begin{abstract}
A large-scale circulation, a turbulent boundary layer, and a turbulent plume are noted features of convection at large Rayleigh numbers under differential heating on a single horizontal boundary. These might be attributed to the forcing, which in all studies has been limited to a unidirectional gradient over the domain scale. We instead apply forcing on a length scale smaller than the domain, and with variation in both horizontal directions. Direct numerical simulations show turbulence throughout the domain, a regime transition to a dominant domain-scale circulation, and a region of logarithmic velocity in the boundary layer, despite zero net heat flux. The results show significant similarities to Rayleigh-Bénard convection, demonstrate the significance of plume merging, support the hypothesis that the key driver of convection is the production of available potential energy without necessarily supplying total potential energy, and imply that contributions to domain-scale circulation in the oceans need not be solely from the large-scale gradients of forcing.
\end{abstract}

DOI: 10.1103/PhysRevLett.115.204301

PACS numbers: 44.25.+f, 47.27.E-, 47.55.pb

The complex dynamics of convection, turbulence, and heat transport in Rayleigh-Bénard convection, where a temperature difference is applied between the top and bottom of a layer of fluid, continue to challenge modern physics [1-3]. Under strong forcing the convection is turbulent and a particular problem has been to understand the cause and consequences of a large-scale circulation, or "wind" [4-6], and turbulent dissipation [7].

In recent direct numerical simulations (DNS) of Rayleigh-Bénard convection [7] the roles of large and small scales of motion were separated, showing quantitatively for the first time that under stronger forcing the flow and heat transport are increasingly dominated by large-scale, longlived flow structures. (By strong forcing we mean $\mathrm{Ra}>10^{9}$, where $\mathrm{Ra}=g \alpha \Delta T H^{3} / \kappa \nu$ is the Rayleigh number, with gravitational acceleration $g$, applied temperature difference $\Delta T$ across the fluid depth $H$, and the fluid thermal expansion coefficient $\alpha$, diffusivity of heat $\kappa$, and kinematic viscosity $\nu)$. Large-scale winds release gravitational potential energy (PE) and transport heat more rapidly than do the smaller scales. At the same time the wind undergoes shear instability, transferring kinetic energy (KE) into small scales of turbulence. Although the dissipation rate is a maximum in the boundary layers, as measured [8,9], the DNS [7] shows that almost all dissipation occurs throughout the interior (bulk) of the flow. The net (volume integrated) production of $\mathrm{KE}$ and the dissipation rate scale as NuRa $[1,7,10]$ and, hence, approximately as $\mathrm{Ra}^{1.3}$ (utilizing the heat transport result $\left.\mathrm{Nu} \sim \mathrm{Ra}^{0.3}[6,11]\right)$. The wind is the main source of shear production, allowing greater turbulent dissipation. It emerges in this system despite perfectly uniform boundary conditions and is qualitatively understood to involve a sweeping up of many small-scale plumes at the boundaries into larger, more efficient structures.
Convection also occurs when a temperature difference is applied on a horizontal boundary along the length of a box of fluid [12-14]. The resulting "horizontal convection" may, for example, contribute significantly to global overturning of the oceans [15-20]. Once in thermal equilibrium this kind of convection carries no net heat transport through any horizontal level and the forcing boundary conditions impose a horizontal length scale, which is realized as the dominant scale in the flow. Laboratory experiments reveal small-scale convective instabilities in limited regions $[14,21]$ becoming turbulent for $\mathrm{Ra}_{L} \sim 10^{12}$ (in this case the forcing parameter, $\mathrm{Ra}_{L}$, is defined using the horizontal scale of forcing $L$ in place of $H$ [12]). Simulations show turbulent statistics [22] at $\mathrm{Ra}_{L} \sim 10^{11}$ and transitions from viscous to inertial regimes [13] commencing at $\operatorname{Ra}_{L} \sim 10^{10}$, not fully complete in terms of the energetics at $\operatorname{Ra}_{L} \sim 10^{15}$.

The turbulent solutions were explained [23], as the dissipation, or net production of $\mathrm{KE}$, is actually the difference between two much larger conversions: a large production of KE [representing conversion of the available potential energy (APE) [24] produced by the boundary buoyancy forcing [17]] in a plume at one end of the box (equivalent to localized deep sinking at high latitude locations in the oceans), but a continual conversion of $\mathrm{KE}$ back into APE in other regions (where there is slow vertical flow despite stable density stratification). Scaling theory and DNS show that the production of APE by the boundary thermal forcing, hence, gross KE production, increases nonlinearly as $\mathrm{Ra}_{L}^{13 / 10}$ [13] (similar to the result for the Rayleigh-Bénard convection, where APE and net KE production scale together [7]). Thus, in horizontal convection for any $\mathrm{Ra}_{L}>10^{9}$ gross KE production far outstrips the net (which scales only linearly as $\mathrm{Ra}_{L}$ ), 
leaving viscous dissipation accounting for a negligible fraction of the APE production. (Almost all of the APE is instead converted into background PE through irreversible mixing $[13,23,25])$. These observations led to the hypothesis $[13,23]$ that dissipation plays at best a secondary role and is not a useful indicator of the rate of the circulation.

All previous studies of horizontal convection have been limited by two conditions: the temperature difference applied on the boundary was unidirectional and on the scale of the domain (like a meridional gradient at the ocean surface), which together force a dominant two-dimensional circulation over the length of the domain, and a single plume into which the KE generation and shear production are focused. We remove both constraints in order to examine the implications for the scales of motion, development of turbulence, and role of dissipation. DNS was carried out for a layer of depth $H$ in a square domain of width $W$ above a planar horizontal boundary heated and cooled in a square array (Fig. 1 bottom plane). The boundary temperature was prescribed as $T=\Delta T \sin ^{2}(\pi x / L) \sin ^{2}(\pi y / L)$, where $(x, y)$ are horizontal coordinates, $\Delta T$ is the temperature difference, $L$ is the wavelength of the pattern, and $n$ is the number of wavelengths within the computational domain $0 \geq(x, y) \geq n L$. The forcing scale $L$ and domain width $W=n L$ for our investigation would ideally be vastly different. However, we also focus on large $\mathrm{Ra}_{L}$, for which the computational demand, given lengthy integrations to thermal equilibrium [21], restricts us to $n \leq 4$, or up to 16 hot patches. The flow is fully defined by four dimensionless parameters: $\operatorname{Ra}_{L}, n, H / L$, and the Prandtl number $\operatorname{Pr}=\nu / \kappa$. Solutions were computed for $\operatorname{Pr}=1$ with $n=3$ and 4 , $H / L=0.5$ and 5 , and $\mathrm{Ra}_{L}=10^{12}$.

The DNS solves the Navier-Stokes and continuity equations in the incompressible, Boussinesq approximation with a linear density-temperature relation, as previously described and tested for both the Rayleigh-Bénard and horizontal convection $[7,13]$. A pseudospectral approach was used for most solutions, with periodic boundary conditions on all lateral boundaries, impermeable and no-slip boundaries at the top and bottom, and adiabatic at the top. A more demanding simulation was carried out for impermeable sidewalls (with $n=4, H / L=5$ ) using finite differences in all directions. The solutions rigorously satisfy grid resolution and time step criteria, along with grid convergence, mechanical energy and heat budget tests [7,13]. Each case was initiated with a uniform fluid temperature and allowed to evolve in time until it reached thermal equilibrium indicated by no long-term secular trend and vanishing net heat transport through the bottom boundary. Flow statistics describing the roles of large and small length scales are estimated by decomposing the velocity field and mechanical energy budget at each location into a time mean (windowed over three buoyancy time scales and equivalent to large length scales)

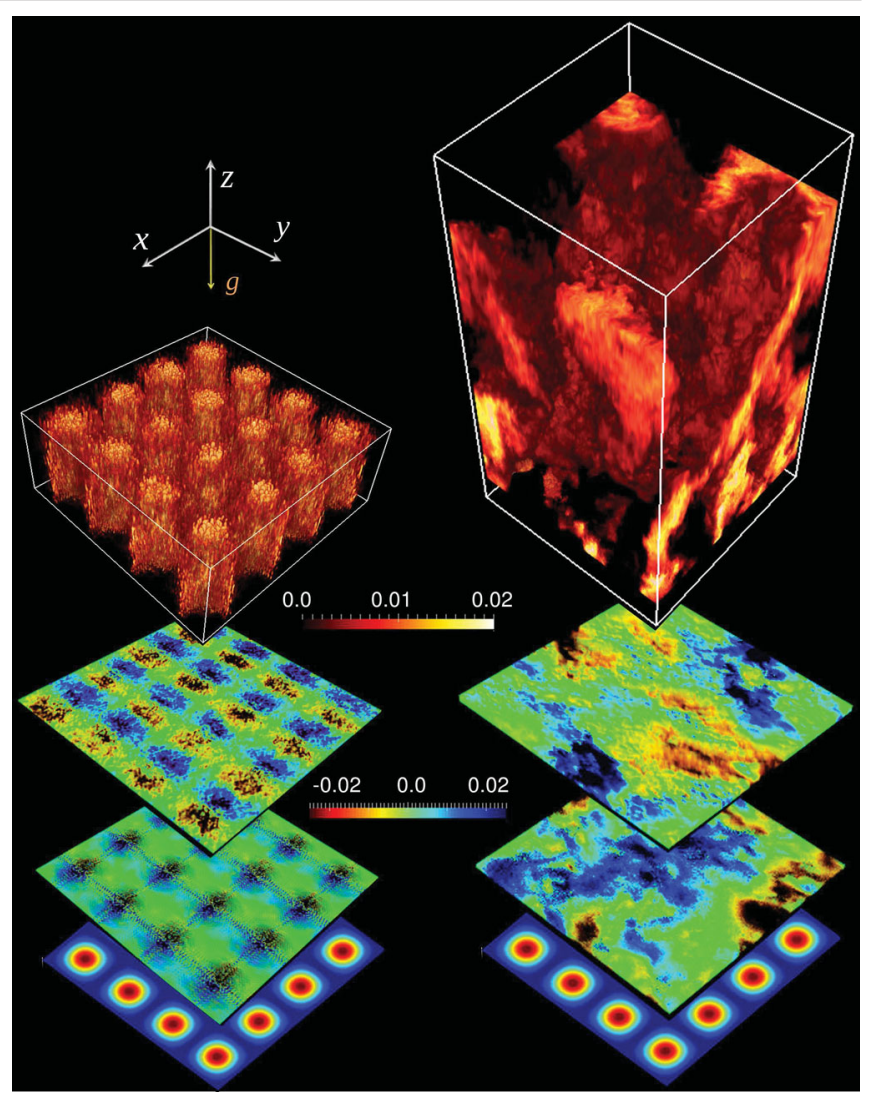

FIG. 1 (color). Snapshots of DNS solutions for $n=4$ and $\operatorname{Ra}_{L}=10^{12}$ : on the left a shallow layer $(H / L=0.5)$, on the right a deep layer $(H / L=5)$. From the bottom: temperature distribution imposed on the boundary (red: hot, blue: cold), horizontal sections showing velocity component $u$ in the $x$ direction at $z / H=0.04$ (just above the bottom boundary layer) and $z / H=$ 0.5 (mid-depth), and, at the top, 3D maps of vertical velocity $w$ (shown only where $w$ is positive, black where negative, with the solution in the upper $10 \%$ of the domain removed to reveal $w$ on the plane, $z / H=0.9)$. Scales show $u$ and $w$ normalized by $(g \alpha \Delta T H)^{1 / 2}$.

and fluctuating components of higher frequencies (smaller length scales), following recent methodology for RayleighBénard convection [7].

For the shallow layer $(H / L=0.5)$ (Fig. 1, left panel) the convection forms an array of discrete, coherent plumes in which the fluid ascends vertically above each boundary hot patch. Remarkably, despite zero net heat flux, the plumes are turbulent and expand with height as a result of turbulent entrainment of the surrounding fluid; fluid between the plumes descends. The plumes persist in the stable array, indicating that inertial interactions between them are weak. The flow is qualitatively like that in previous studies with one-dimensional boundary forcing and a single line plume $[13,14]$, accepting that in the present case there is radial convergence of flow into each plume.

In the deep layer $(H / L=5)$ with $n=3$, the array of coherent plumes again remains stable in thermal equilibrium. However, for $n=4$ [Fig. 1, right panel, and Fig. 2(a)] 
(a)
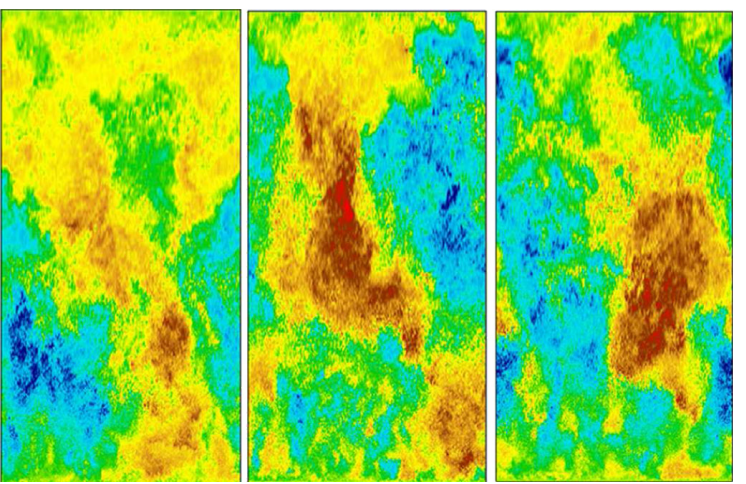

(b)
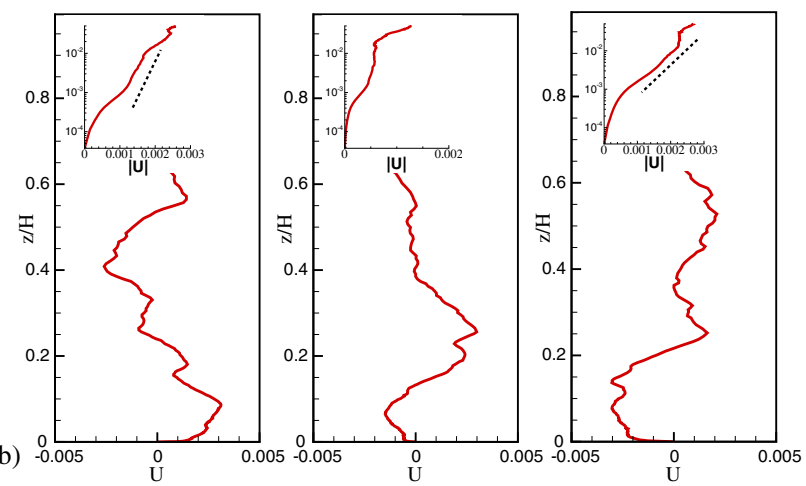

FIG. 2 (color online). The velocity field for the deep-layer case $(H / L=5)$. (a) Vertical velocity $w$ on a vertical plane at $x / L=$ 1.875 (which passes through a row of hot spots on the bottom boundary), shown at three instants separated by eight buoyancy time scales $[H /(g \alpha \Delta T)]^{1 / 2}$. Large scales of vertical exchange and boundary layer sweeping are dominant. (b) Instantaneous vertical profiles of horizontal velocity $u$ averaged over the horizontal area of the domain at each height $z$, for the same times as (a). These illustrate a reversal of the mean horizontal wind. Insets show on $\log$-log scales the depth dependence of the magnitude $|u|$ of the horizontal velocity in the bottom $10 \%$ of the domain. The velocity boundary layer extends from the bottom to $z \approx 0.01 \mathrm{H}$ with a logarithmic dependence (slope of dotted lines) through more than $90 \%$ of the boundary layer during active phases of the large-scale wind.

individual warm plumes are seen only intermittently. Large-scale sweeping motions tend to carry the warm fluid away from the heated patches of the base, collecting it into one or two updrafts. These large updrafts are of similar size and speed as the downdrafts, an obvious difference from the highly asymmetric structure of vertical motions in previous horizontal convection studies. Further, these domain-filling overturns are unsteady and occasionally reverse on time scales long compared with the typical time for fluid to be carried around the domain [Fig. 2(b)]. The bottom boundary layer has a logarithmic profile of the horizontal velocity during phases of maximum mean wind velocity [Fig. 2(b), left and right panels], strong evidence that the boundary layer is turbulent as a result of local shear instability. The logarithmic velocity structure disappears at times when the wind is reversing [Fig. 2(b), middle panel].
The flow field is also characterized by a full spectrum of length scales throughout the interior: the kinetic energy spectrum (Fig. 3) shows the $-5 / 3$ power law expected for an inertial turbulence range over at least 2 orders of magnitude in wave number (for the deep layer), and a steeper dissipation range at small scales extending to the Kolmogorov scale. Notably, the energy spectrum in this deep-layer case has a maximum at the domain scale, $4 L$, rather than at the forcing scale.

Vertical profiles of temperature, turbulent dissipation rate $\left(\varepsilon_{\text {turb }}\right)$, production of turbulent kinetic energy by shear instabilities $(P)$, and APE-to-turbulence KE conversion $\left(B_{\text {turb }}\right)[7,13]$ for the deep-layer case are shown in Fig. 4. Shear production and turbulent dissipation are similar and fairly uniform with height, and an order of magnitude larger than buoyancy flux, as turbulence is produced mainly by shear instability rather than convection. Thus, turbulence production and dissipation are almost entirely outside the thin boundary layer.

The simulations show also that the total viscous dissipation over the volume of the domain is achieved predominantly through small-scale turbulence. In the solution of Figs. 3 and 4, which has periodic side boundaries, the total dissipation is approximately 3 times larger than the production $\Phi_{i}$ of potential energy (which is by molecular diffusion of heat down the stable average vertical temperature gradient between the top and bottom of the fluid), whereas the solution with impermeable sidewalls shows total dissipation exactly equal to $\Phi_{i}$, as theoretically

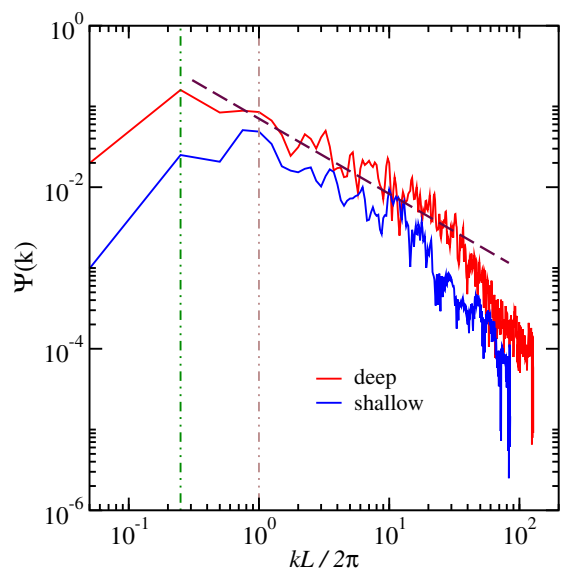

FIG. 3 (color online). Kinetic energy power spectra [in dimensionless form after normalizing velocity by $(g \alpha \Delta T H)^{1 / 2}$ ] as a function of horizontal wave number $k$ (normalized by the wave number $2 \pi / L$ of the forcing) for $n=4, \mathrm{Ra}_{L}=10^{12}$ : deep layer (red line), shallow layer (blue line). Data are averages over 20 distributed locations in the interior of the flow. Vertical dotted lines show the width of the domain at $2 \pi / L=0.25$ and thermal forcing scale at $2 \pi / L=1.0$; the Kolmogorov wavelength at the end of the dissipation range is at $2 \pi / L=3 \times 10^{2}$ and $1.5 \times 10^{2}$ for the deep and shallow layers, respectively. A $-5 / 3$ slope (broken line) describes the results in the deep layer for $0.25<k L / 2 \pi<30$. 

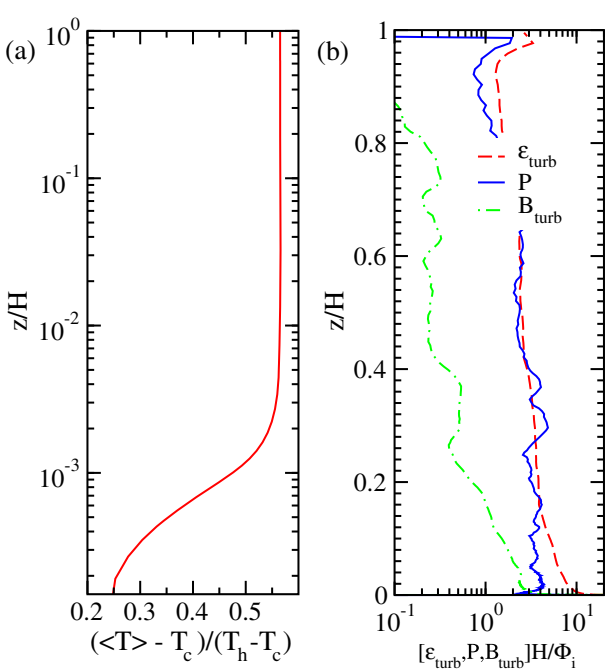

FIG. 4 (color online). Vertical profiles of (a) normalized temperature and (b) normalized rates of energy conversion $P$, $\varepsilon_{\text {turb }}$ and $B_{\text {turb }}$. All quantities are horizontal averages over the area of the domain at each height. Temperature, relative to the coldest boundary temperature $\left(T_{c}\right)$ and normalized by $\Delta T$, is shown on a logarithmic height axis, revealing the thin boundary layer. Energy conversions, nondimensionalized by the domain total rate of generation $\left(\Phi_{i}\right)$ of potential energy divided by fluid depth $(H)$ $[7,23]$, are plotted on a linear height axis.

required [26]. Vertically periodic boundary conditions have also been shown to promote inertia-dominated dynamics in Rayleigh-Bénard convection [27]. However, in the present system the impermeable boundary solution shows that the turbulent nature of the flow is not dependent on the use of periodic side boundary conditions.

In contrast, the production of APE is very much larger than both the dissipation rate and production of PE (by $10^{3}$ times for the deep layer with periodic side boundaries, a factor 2 orders of magnitude larger than was found for the usual horizontal convection problem at the same $\mathrm{Ra}_{L}$ [23]). The full mechanical energy cycle is explained as for horizontal convection $[10,13,23]$ : the APE arises through conversion from background $\mathrm{PE}$ by the horizontal differences in boundary heat flux and it is released to tge KE of either the upwelling plumes (shallow layer) or the large-scale circulation (deep layer), before most of the KE is returned to APE by vertical advection against the stable interior density gradient elsewhere in the domain, and ultimately returned to background PE by diffusion of heat in the thin boundary layer on the base.

Thus, coherent turbulent plumes dominate the flow in the shallow layer case as a consequence of a supply rate of APE that is very large compared with negligible rates of PE production and KE dissipation. Still, greater production of APE in the deep layer owing to the larger height, likely coupled with as yet unexplored dependence on the aspect ratio $n=W / L$, leads to plume interactions and regime change to a strong dominance of domain-scale motions.
Note that the present flow is conceptually like eight horizontal convection boxes placed end to end in each direction, each having the value of $\mathrm{Ra}_{L}$ used here; in the shallow layer case the eight end wall plumes become four fixed turbulent plumes, whereas in the deep-layer case the plumes merge into a single, stronger large-scale updraft that causes much greater shear production. The strength of the large-scale circulation and turbulent nature of the flow despite zero net buoyancy flux and negligible PE production support the hypothesis $[17,23,25]$ that APE production by boundary buoyancy forcing is the key driver in the physics of convection more generally. The multiple similarities between the deep-layer case and Rayleigh-Bénard convection (at $\mathrm{Ra}>10^{10}$ [7]) include a large role of shear instability, an inertial cascade of energy down to small scales overwhelmingly in the interior, a logarithmic profile in the boundary layer [28], and a dominance of the longlived domain scales of motion in the mechanical energy budget and transport of heat. On the other hand, the significance of turbulent dissipation in the two kinds of convection is very different, amounting to one half of the APE released in Rayleigh-Bénard convection [10,25] but to only a small term in the (even the net) mechanical energy budget when heating and cooling is at the same horizontal boundary.

The small-scale forcing geometry is conceived for the purposes of revealing aspects of the dynamics beyond those seen under a domain-scale boundary temperature gradient. It shows that sweeping of the boundary layer and merger of plumes is a key to the formation of large-scale, more efficient circulation despite greater shear and dissipation. Further examination of the parameter dependence and of superposing small- and domain-scale gradients is warranted to assist in a conceptual understanding of the role of convection in the global meridional overturning circulation of the oceans, which is generally seen as a response to the zonally averaged meridional gradient of surface boundary conditions [15-20]. A zonal average overlooks the additional energy input by zonal variations in buoyancy forcing and may underestimate the role of convection.

This research was funded by Australian Research Council Grants No. DP120102744 and No. DP140103706. B. G. was supported by an ARC Fellowship DE140100089. The simulations were made possible by time allocations granted by the Australian National Computational Infrastructure facility.

*Corresponding author.

Ross.Griffiths@anu.edu.au

[1] E. D. Siggia, Annu. Rev. Fluid Mech. 26, 137 (1994).

[2] G. Ahlers, S. Grossmann, and D. Lohse, Rev. Mod. Phys. 81, 503 (2009).

[3] D. Lohse and K.-Q. Xia, Annu. Rev. Fluid Mech. 42, 335 (2010). 
[4] J. W. Deardorff and G. E. Willis, J. Fluid Mech. 28, 675 (1967).

[5] R. Krishnamurti and L. N. Howard, Proc. Natl. Acad. Sci. U.S.A. 78, 1981 (1981).

[6] J. Niemela, L. Skrbek, K. R. Sreenivasan, and R. Donnelly, Nature (London) 404, 837 (2000); J. Fluid Mech. 449, 169 (2001).

[7] B. Gayen, G. O. Hughes, and R. W. Griffiths, Phys. Rev. Lett. 111, 124301 (2013).

[8] B. Puthenveettil and J. Arakeri, J. Fluid Mech. 542, 217 (2005).

[9] R. Ni, S.-D. Huang, and K.-Q. Xia, Phys. Rev. Lett. 107, 174503 (2011).

[10] G. O. Hughes, B. Gayen, and R. W. Griffiths, J. Fluid Mech. 729, R3 (2013).

[11] S. J. A. M. Stevens, D. Lohse, and R. Verzicco, J. Fluid Mech. 688, 31 (2011).

[12] H. T. Rossby, Deep-Sea Res. 12, 9 (1965); Tellus 50A, 242 (1998).

[13] B. Gayen, R. W. Griffiths, and G. O. Hughes, J. Fluid Mech. 751, 698 (2014).

[14] J. C. Mullarney, R. W. Griffiths, and G. O. Hughes, J. Fluid Mech. 516, 181 (2004).
[15] H. Stommel, Proc. Natl. Acad. Sci. U.S.A. 48, 766 (1962).

[16] R. Lumpkin and K. Speer, J. Phys. Oceanogr. 37, 2550 (2007).

[17] G. O. Hughes, A. McC. Hogg, and R. W. Griffiths, J. Phys. Oceanogr. 39, 3130 (2009).

[18] J. Marshall and K. Speer, Nat. Geosci. 5, 171 (2012).

[19] M. Nikurashin and G. Vallis, J. Phys. Oceanogr. 42, 1652 (2012).

[20] J. A. Saenz, A. McC. Hogg, G. O. Hughes, and R. W. Griffiths, Geophys. Res. Lett. 39, L13605 (2012).

[21] R. W. Griffiths, G. O. Hughes, and B. Gayen, J. Fluid Mech. 726, 559 (2013).

[22] A. Scotti and B. White, Geophys. Res. Lett. 38, L21609 (2011).

[23] B. Gayen, R. W. Griffiths, G. O. Hughes, and J. A. Saenz, J. Fluid Mech. 716, R10 (2013).

[24] K. B. Winters, P. N. Lombard, J. J. Riley, and E. A. D' Asaro, J. Fluid Mech. 289, 115 (1995).

[25] A. Scotti and B. White, J. Fluid Mech. 740, 114 (2014).

[26] F. Paparella and W. Young, J. Fluid Mech. 466, 205 (2002).

[27] D. Lohse and F. Toschi, Phys. Rev. Lett. 90, 034502 (2003).

[28] G. Ahlers, E. Bodenschatz, D. Funfschilling, S. Grossmann, X. He, D. Lohse, R. J. A. M. Stevens, and R. Verzicco, Phys. Rev. Lett. 109, 114501 (2012). 